\title{
Frailty, Implantable Cardioverter Defibrillators, and Mortality: a Systematic Review
}

\author{
Michael Y. Chen, $M D^{7}$, Ariela R. Orkaby, MD, MPH 2,3 , Michael A. Rosenberg, $M D^{4,5}$, and
}

Jane A. Driver, $M D, M P H^{2,3}$

\begin{abstract}
'SUNY Downstate, Brooklyn, NY, USA; ${ }^{2}$ New England Geriatric Research, Education, and Clinical Center (GRECC), VA Boston Healthcare System, Boston, MA, USA; ${ }^{3}$ Division of Aging, Brigham \& Women's Hospital, Harvard Medical School, Boston, MA, USA; 'Division of Biomedical Informatics and Personalized Medicine, University of Colorado School of Medicine, Aurora, CO, USA; ${ }^{5}$ Division of Cardiac Electrophysiology, University of Colorado School of Medicine, Aurora, CO, USA.
\end{abstract}

BACKGROUND: Evidence for the benefit of implantable cardioverter defibrillators (ICD) in preventing sudden cardiac death (SCD) in older adults is mixed; age alone may not predict benefit. Frailty may help identify patients in whom an ICD does not improve overall mortality risk.

METHODS: Structured search of PubMed, Embase, Web of Science, and Cochrane Central Register of Controlled Trials on $1 / 31 / 2019$, without language restriction, with terms for ICD, frailty, and mortality. Frailty was defined broadly using any validated single component (e.g., walking speed, weight loss) or multi-component tool (e.g., cumulative deficit index). Each study was assessed for quality and risk of bias.

RESULTS: We identified and screened 2649 titles, reviewed 280 abstracts, and extracted 71 articles. Nine articles, including two RCTs, one prospective cohort, and six retrospective cohort studies met all criteria. The most common reason for exclusion was a lack of frailty definition. Frailty definitions were heterogeneous, including cumulative deficit models, low weight, and walking speed. Follow-up time for mortality differed: from days to > 6 years. All studies indicated that mortality was higher amongst individuals identified as frail, regardless of definition. In one RCT, slow walkers did not benefit from ICD therapy after 3 years. A cohort of 83,792 Medicare beneficiaries in an ICD registry reported higher 1-year mortality following ICD in those with frailty or dementia. Four studies reported an association between being underweight and increased mortality following ICD placement. CONCLUSION: Existing literature suggests that individuals with frailty may not benefit from ICD placement for primary prevention of SCD.

Michael Y. Chen and Ariela Orkaby contributed equally as first authors.

Prior Presentations Chen MY, Orkaby AR, Rosenberg MA, Driver JA. Frailty May Identify Older Patients Who Do Not Benefit From Implantable Cardioverter Defibrillators: a Systematic Review. Poster: American Geriatric Society 2017 Annual Meeting, San Antonio, TX. Poster \# D150.

Electronic supplementary material The online version of this article (https://doi.org/10.1007/s11606-019-05100-9) contains supplementary material, which is available to authorized users.

Received February 19, 2019

Revised March 8, 2019

Accepted May 13, 2019

Published online July 1, 2019
KEY WORDS: frailty; implantable cardioverter defibrillator; sudden cardiac death.

$\mathrm{J}$ Gen Intern Med 34(10):2224-31

DOI: $10.1007 /$ s11606-019-05100-9

(C) Society for General Internal Medicine (This is a U.S. government work and not under copyright protection in the U.S.; foreign copyright protection may apply) 2019

\section{INTRODUCTION}

Implantable cardioverter defibrillators (ICD) therapy remains the most effective preventive strategy for sudden cardiac death (SCD). In the USA, more than 350,000 patients experience SCD annually. ${ }^{1,2}$ For primary prevention of SCD, current American College of Cardiology/American Heart Association guidelines recommend ICD therapy for patients who are at risk of a life-threatening ventricular arrhythmia but have already received optimal medical management. However, benefit of ICD therapy for primary prevention in older individuals remains unclear. ${ }^{3-5}$ Current US guidelines do not specify a upper age limit for ICD placement. ${ }^{6}$ The broad nature of current guidelines leaves room for physician discretion, asking practitioners to determine that the patient has "reasonable expectation of survival for at least a year". ${ }^{7}$ Life expectancy can be challenging to assess for many older individuals when chronologic age can be a poor marker of a measure of biologic age. ${ }^{8}$ Furthermore, current directives from the Centers for Medicare and Medicaid require that physicians engage in shared decision-making conversations regarding risks and benefits before ICD placement for primary prevention. ${ }^{9,10}$ Adding to this challenge is the conflicting evidence on the relationship between patient age and survival after ICD placement. ${ }^{11,12}$ Between the ages of 70 and 90 , in which there is a wide variation in health status, measures of frailty are often more accurate predictors of death than age itself. ${ }^{13,14}$

Frailty is defined as a state of increased vulnerability and poor resolution of homeostasis following a stressor, which increases the risk of adverse outcomes including hospitalization, institutionalization, and mortality. ${ }^{13}$ This increased vulnerability may be related to malnutrition or immunosuppression. A narrative review in 2016 suggested that frailty is a major risk factor for death and readmission in patients who received ICD for both 
primary and secondary prevention of SCD. ${ }^{15}$ We sought to update this synthesis with a systematic review to determine whether frailty identifies older patients who may not benefit from ICD therapy specifically for primary prevention of SCD.

\section{METHODS}

\section{Protocol and Registration}

This systematic review was conducted in accordance with the PRISMA guidelines. ${ }^{16}$ We did not register a protocol for the review.

\section{Eligibility Criteria}

To be included in this review, studies had to include (1) participants $>65$ years who received an ICD for primary SCD prevention, (2) any accepted definition of frailty, and (3) mortality as an endpoint. Studies that reported only secondary prevention or defibrillators with cardiac resynchronization therapy (CRT-D) were excluded. We did not impose any restriction on the type of study, publication date, or publication status for the search. We excluded non-English studies during screening of abstracts.

\section{Defining Frailty}

Although there is no consensus definition, there are currently two leading theories of frailty: the phenotype model defined by Fried and colleagues and the cumulative deficit model developed by Rockwood and colleagues. ${ }^{14,17}$ The phenotype model defines frailty as meeting three or more of five criteria: weight loss, exhaustion, weakness (grip strength), slow walking speed, and low physical activity. ${ }^{14}$ The cumulative deficit model assesses frailty by summing health-related deficits associated with aging that occur over the lifespan, including morbidity, function, mood, cognition, and disability. ${ }^{17}$ In addition to these two theories, there are multiple definitions that include a single component (e.g., gait speed, weight loss), or multiple components (e.g., comprehensive geriatric assessment, allostatic load) ${ }^{18}$ In order to include as many articles as possible in this review, we built our search string to capture any of the currently used definitions of frailty, from the single component to complex multi-component definitions, as has been recommended in other systematic reviews that included the term frailty. ${ }^{18}$ Low BMI was included as a proxy for weight loss or poor nutrition. ${ }^{19}$

\section{Information Sources}

Studies were identified by searching electronic databases and scanning reference lists of articles. The search was applied to MEDLINE, Embase, Web of Science, and the Cochrane Central Register of Controlled Trials on January 31, 2019.

\section{Search}

The search string was constructed with the help of an experienced medical librarian using three components: frailty, ICD, and mortality. Terms representing frailty such as hand or grip strength, gait speed, mobility, weight loss, and functional impairment were used. Further details on the search string used are available in the appendix.

\section{Study Selection}

Eligibility assessment was performed independently in an unblinded standardized manner by two reviewers. Disagreements between reviewers were resolved by consensus. Titles, abstracts, and articles were screened by two reviewers (ARO, MYC) with an additional reviewer to resolve discrepancies (JAD) using Endnote X7. Information was extracted from each included study on (1) type of article, (2) study question, (3) characteristics of participants: population, age, number of participants, (4) frailty definition, and (5) mortality definition: peri-operative, in-hospital, and cause.

\section{Level of Evidence}

The level of evidence was determined by (1) study design: a randomized controlled trial or a cohort study, (2) any frailty definition, (3) generalizability, and (4) bias: statistical power, conflict of interest, and validity of methods. The quality of studies was appraised using the Newcastle/Ottawa Scale (NOS). Criteria for quality assessment are shown in Appendix Fig. 2.

\section{RESULTS}

The selection process for the articles included in this review is illustrated in Appendix Fig. 1. The database search yielded a total of 2649 records. Of these, 574 duplicate records were excluded. In total, 280 abstracts were identified for screening, and 71 articles were selected for full-text assessment. Nine articles were identified for final inclusion in the review, including two randomized controlled trials, six retrospective cohort studies, and one prospective cohort study. Measures of frailty included a variation of the cumulative deficit model (1 study), low physical activity (3 studies), weight loss/low BMI (3 studies), allostatic overload (1 study), and advanced age with comorbidity (1 study). Follow-up time for mortality differed in each study: from 1 day to 6 years. Sample size also varied across studies with smallest sample of 77 and largest sample of 98,437 (Table 1). Meta-analysis could not be performed due to the significant differences amongst studies in frailty definition and mortality outcomes.

The two RCTs included both used a marker of physical activity to assess frailty and found that low physical activity, either by self-report or measured was associated with increased risk of mortality. Piotrowicz and colleagues administered the Short Form-12 Health Survey to participants in the Multicenter Automatic Defibrillator Implantation Trial II (MADIT-II) measuring baseline physical function prior to ICD placement and at subsequent follow-ups. Participants 


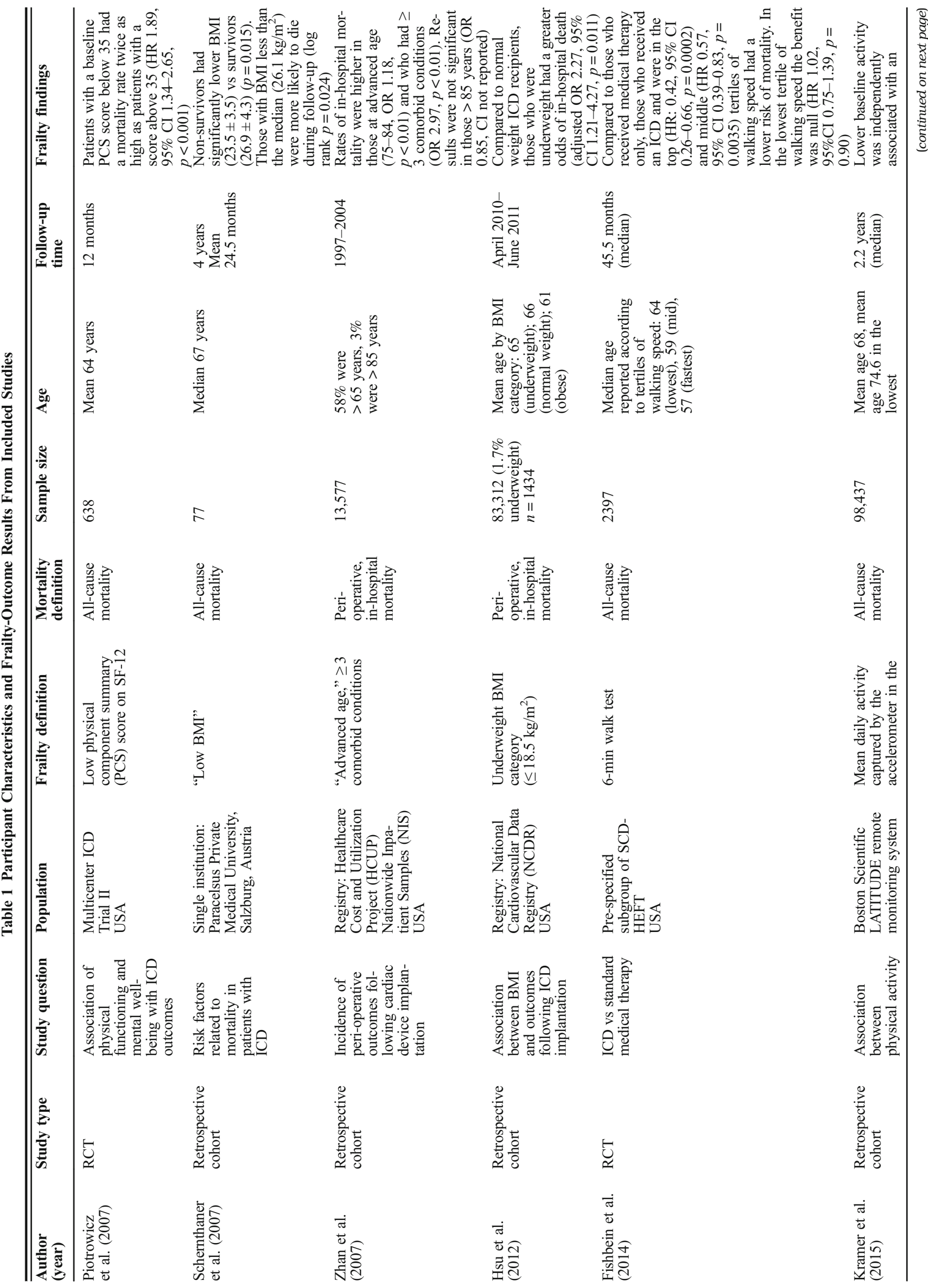




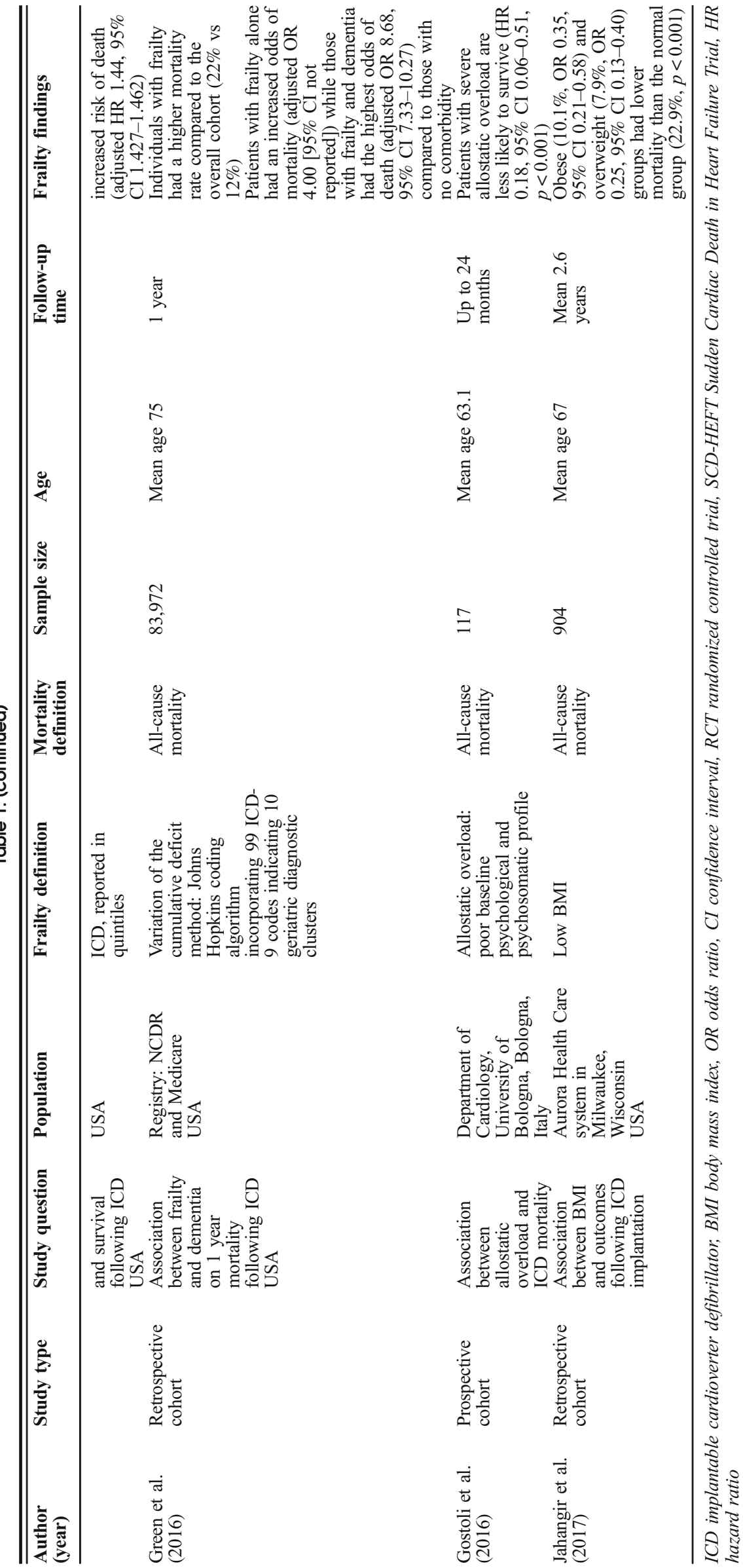


with a baseline physical component score below the median 35 were nearly twice as likely to die (HR 1.89, 95\% CI 1.34$2.65)$ as those with a score above the median. ${ }^{20}$ Fishbein et al. used the 6-min walk test (6MWT) to assess function and predict survival following ICD placement in a pre-specified subgroup of the Sudden Cardiac Death in Heart Failure Trial (SCD-HEFT). Risk of mortality amongst those with ICD vs placebo was assessed by tertile of $6 \mathrm{MWT}$ at baseline. In the highest tertile, all-cause mortality was $7 \%(n=28)$ vs placebo $17.8 \%(n=60)$, compared to $12.4 \%(n=44)$ vs placebo $21.6 \%$ $(n=78)$ in the middle tertile, and $28 \%(n=92)$ vs placebo $24.7 \%(n=83)$ in the lowest tertile. Every 50-m improvement in 6MWT distance was associated with an adjusted hazard ratio of 0.88 (95\% CI $0.81-0.96)$. Overall, ICD therapy substantially benefited patients who were in the top two tertiles of 6MWT distance but not patients from the lowest tertile, a marker of frailty. ${ }^{21}$

In a cohort study that used physical activity as a marker of frailty, results were similar. Kramer et al. reported that mean baseline activity was inversely correlated with mortality in a cohort of 98,437 US patients followed for a median of 2.2 years after ICD placement. Baseline activity was defined as mean minutes per day considered active from day 30 to day 60 after ICD implantation. A decrease of 30-min baseline activity was correlated with a $48 \%$ increased hazard ratio for death. Mean 4-year survival was much lower in the least active quintile compared to the most active quintile ( 0.76 vs 0.91$){ }^{22}$

In cohort studies that used low BMI or weight loss as a marker of frailty, low BMI was associated with a higher mortality rate, although no consensus on the cutoff of BMI was given. Schernthaner et al. conducted a retrospective cohort study including 77 patients who received ICDs in Austria with a median age of 67 , followed for a mean 24.5 months and concluded that $\mathrm{BMI} \leq 26.0 \mathrm{~kg} / \mathrm{m}^{2}$ is an independent predictor of mortality $(p=0.024) .^{23}$ Similarly, Jahangir and colleagues found that the presence of obesity or overweight BMI was associated with lower post-ICD mortality compared to those with normal BMI group in a group of patients in Wisconsin. ${ }^{24}$ Hsu and coworkers used US national cardiovascular registry data to examine outcomes for 83,312 patients and found that those who were underweight $\left(\mathrm{BMI} \leq 18.5 \mathrm{~kg} / \mathrm{m}^{2}\right)$ had more than twofold higher odds of in-hospital death comparing to normal weight patients. ${ }^{25}$

Zhan and colleagues defined frailty as advanced age and the presence of comorbidities and reported that the inhospital mortality rate following ICD placement was low, at $0.75 \%$, consistent with other types of cardiac device implantation, including CRT-D (0.93\%), CRT-P (1.40\%), and pacemakers $(1.12 \%)$. However, amongst those who received an ICD and were frail, there was an increased risk of in-hospital mortality compared to those with no comorbid conditions (OR 2.97, $p<0.01$ ). For those who were aged 75-84 the odds ratio for in-hospital mortality was $1.18(p<0.01)$. However, the OR for those $>85$ was not significant at $0.85 .^{26}$
Green and colleagues used the Johns Hopkins Adjusted Clinical Group (ACG) System, a tool that analyzes health insurance claims and enrollment records to predict a person's future healthcare utilization, as their definition of frailty. The ACG System frailty markers included 10 categories: falls, difficulty walking, social support needs, weight loss, fecal incontinence, urine incontinence, decubitus ulcer, severe vision impairment, dementia, and malnutrition. The study reported that 1-year mortality following ICD placement was $22 \%$ for patients with frailty but only $12 \%$ in the overall cohort. $^{27}$ Using baseline psychological and psychosomatic profile, Gostoli and colleagues found that traditional psychological risk factors such as depression, anxiety and type-D personality are not good predictors of post-ICD mortality. However, patients with severe allostatic overload, or the inability to cope with stress, are less likely to survive (HR 0.18 , $95 \%$ CI $0.06-0.51, p<0.001) .^{28,29}$

\section{DISCUSSION}

In this systematic review, we found that frailty, defined according to varying validated methods, was consistently associated with higher risk of mortality after ICD placement. Six of the nine studies included used a single marker of frailty, while three studies used multi-domain instruments. The main finding of this systematic review is that older patients who had measures associated with frailty and received an ICD for primary prevention of SCD had a higher mortality risk than those who were not frail. Although assessment of frailty varied, the main finding remained consistent throughout all nine studies.

Our findings are also consistent with literature in other highrisk cohorts of older adults, such as those with chronic diseases. Chronic diseases and frailty are inseparable under Rockwood's cumulative deficit approach to frailty. Chronic diseases contribute to frailty by decreasing a person's physiological reserve. ${ }^{17} \mathrm{~A}$ recent study by Bansal et al. examined outcomes in 5877 matched patients with an average age of 73 . This study found that ICD placement was not associated with improved survival in patients with chronic kidney disease (adjusted hazard ratio, 0.96; 95\% CI 0.87-1.06) but rather ICD placement was associated with an increased risk of hospitalization due to heart failure (adjusted relative risk, 1.49; 95\% CI 1.33-1.60) and all-cause hospitalization (adjusted relative risk, $1.25 ; 95 \%$ CI $1.20-1.30) .{ }^{30}$ Frailty status was not evaluated in this study.

The ideal frailty instrument remains elusive, and dozens of tools are currently in wide use in the literature. ${ }^{13}$ Given the heterogeneous definition of frailty in the current literature and the search for the most efficient and accurate screening tool for frailty, there is an ongoing debate as to the value of a single component instrument versus multi-domain instrument when designing a standardized quantitative method for measuring frailty. ${ }^{31}$ Every instrument examined in our systematic review 
has its advantages and limitations, and not all meet the classic definitions of frailty. Physical activity can be surveyed or observed. However, accurate measurement of physical activity can be affected by response bias and the Hawthorne effect: participants may under-report or over-report their physical activity on a survey and act differently when knowing that they are being observed. The 6MWT is a popular measure of functional status often used to assess a patient's response to medical intervention. It is typically performed on a $30-\mathrm{m}$ walk course and requires technicians who are well-trained. Results may differ due to factors such as technician and encouragement. ${ }^{32}$ A reasonable surrogate for the $6 \mathrm{MWT}$ is gait speed, which highly correlates with the $6 \mathrm{MWT} .{ }^{33}$ Gait speed similarly suffers some of the limitations of 6MWT but requires shorter walk and has better retest reliability. ${ }^{34}$ In the case of Kramer and coworkers, baseline activity was recorded via the ICD device, which may not be helpful in determining a patient's ICD eligibility prior to implantation. However, slow walking speed is highly correlated with risk of mortality, has been shown to be an excellent screen for frailty, and has been advocated as an ideal single marker of frailty and can be relatively simple to incorporate into a busy clinical setting. ${ }^{35}$

Weight is a quick and simple snapshot of a patient's physical status, but BMI does not account for body composition, genetics, or rate of weight change and may not be an adequate marker of frailty. ${ }^{36}$ The studies examined arbitrarily used the median or the World Health Organization (WHO) cutoffs to categorize their participants. One recurrent finding amongst the studies included in this review was that obesity did not negatively impact mortality rate. This may be explained by the J-shaped paradox of BMI. ${ }^{37}$ Obese patients may have more stable hemodynamic status, more muscle and fat mass, and therefore more energy reserves to resist poor outcomes, whereas low BMI may indicate frailty, poor nutritional status, or decreased immune functions. ${ }^{37}$ Nevertheless, the combination of obesity and decreased muscle mass or strength, a condition known as sarcopenic obesity, may also expose patients to adverse outcomes. Because the three studies that measured BMI did not measure muscle mass, we were not able to determine if patients had sarcopenic obesity. ${ }^{38}$

Allostatic load and the ACG System were multi-domain instruments that incorporated elements of geriatric assessment. Allostatic load is a concept of cumulative wear and tear on the body for survival proposed by McEwen in $1998 .^{39}$ It has been used as an index of multisystem physiological dysregulation including cardiovascular, metabolic, inflammatory, and neuroendocrine systems to predict frailty development. ${ }^{28}$ The operational definition of allostatic overload used by Gostoli and coworkers was developed by Fava and colleagues and focused on overwhelming stressors and clinical manifestations of distress. ${ }^{40} \mathrm{~A}$ detailed interview of stressful life events is required to fully assess an individual's mental health and therefore may not be a time-efficient instrument to assess frailty.

Related to the cumulative deficit model of frailty, Green and colleagues used an algorithm to identify ICD-9 codes from the
ACG System that correspond to geriatric conditions. Of interest, Green et al. emphasized that dementia alone significantly increases mortality risk. Zhan and coworkers defined frailty as a construct of advanced age with comorbidities. Although this deviates from the classic cumulative deficit model of frailty, it is what the authors chose as their definition of frailty. While those with multi-morbidity had a clearly increased risk of inhospital mortality, the role of advanced age was unclear. In recent years, it has become clear that although the risk of frailty rises with age, frailty is a better predictor than age alone and is often independent of age.

To our knowledge, this is the first systematic review specifically evaluating the impact of frailty on mortality in older patients who have undergone ICD implantation for primary prevention of sudden cardiac death. One narrative review, published in 2016, examined the implications of frailty in elderly patients with general electrophysiological conditions, including the need for ICDs. ${ }^{15}$ Bibas et al. concluded that frail patients tend to fare far worse than nonfrail patients who undergo ICD and CRT therapies for both primary and secondary prevention of SCD. ${ }^{15}$ Our systematic review updates this narrative review by performing a systematic literature search using a broad definition of frailty, focusing specifically on ICDs for primary prevention of SCD. This allowed us to include 8 additional studies, 2 of which were published after the prior review.

The benefit of frailty assessment in patient selection for cardiac intervention has been widely recognized in the last few years, including determining eligibility for left ventricular assist device, and for routine cardiovascular care generally. ${ }^{41}$, ${ }^{42}$ However, increased screening for frailty also raises important unanswered questions, particularly when deciding whether to replace or deactivate a patient's ICD, balancing quality of life and lifespan. ${ }^{43}$ Our review addresses this knowledge gap in patient selection for ICD placement and demonstrates the importance of considering aging syndromes, such as frailty, to identifying older individuals who may not benefit from ICD placement. Ongoing efforts in validating frailty as a risk factor for ICD placement include the FRAIL-D study and the Safety of Implantable Cardioverter Defibrillator (ICD) Implantation in the Elderly trial to be completed 2020.

Our study has several limitations. Non-English articles were excluded during the screening process and could have contributed to the limited number of relevant studies found. Not all selected studies solely examined the impact of frailty on mortality in older patients undergoing ICD placement; studies also examined therapies such as CRT, CRT-D. Because ICD was not the main objective in several studies, important ICD patient characteristics could not be isolated.

Seven of the nine studies included are cohort studies and only two randomized controlled studies. All nine studies were published within the last 12 years. Although we have constructed our frailty criteria based on prevailing models of frailty and similar systematic reviews that examine frailty and other cardiac interventions, it is difficult to capture all 
relevant studies because of the elusive definition of frailty. Chronic conditions that could render an individual frail, such as dementia, COPD, and chronic kidney disease, were not part of the search criteria. Green et al. found that approximately $10 \%$ of Medicare patient who received ICD for heart failure in their study population had dementia or frailty. ${ }^{27}$ However, not all chronic conditions lead to frailty; disentangling frailty from comorbidity and disability has been advocated. ${ }^{14}$ Furthermore, although mortality is a standard outcome, because of the varying definitions of frailty that introduce a high degree of heterogeneity we could not perform a meta-analysis of the results, as has been noted in other systematic reviews attempting to synthesize data on frailty and outcomes. ${ }^{18}$

Our study also has several strengths. The article selection process for this review was robust. Relevant electronic databases were included in the search. The search string was constructed based on both Fried's physical phenotype model of frailty and Rockwood's cumulative deficit theory of frailty, as well as individual components and performance measures, as has been done in other systematic reviews of frailty. ${ }^{18}$ This review focused solely on ICD, excluding CRT-D, thus isolating the mortality benefits and risks of defibrillator on patients at risk for sudden cardiac death but not necessarily for heart failure.

\section{CONCLUSION}

In summary, although ICD implantation has been shown to decrease the risk of SCD, in this systematic review, we found that ICD therapy may not benefit all older adults, especially those with frailty. Despite the limited number of studies found, all studies offer evidence that patients who are identified as frail are at increased risk of mortality after ICD implantation. Randomized controlled trials for ICDs that incorporate aging syndromes, such as frailty, are needed to identify which older in older adults will benefit.

Acknowledgments: The authors acknowledged Harvard Librarian, Dr. Paul Bain, for his help with the literature search.

Corresponding Author: Ariela Orkaby, MD, MPH; Geriatric Research, Education, and Clinical Center (GRECC) VA Boston Healthcare System, 150 South Huntington St, Boston, MA 02130, USA (e-mail: aorkaby@bwh.harvard.edu).

Funding Michael Chen: National Institute of Aging/American Federation of Aging Research Medical Scientist Training in Aging Research (MSTAR) grant - NIH/NIA 1T35AG038027 (Driver).

Dr. Orkaby: Veterans Affairs CSRD CDA-2 IK2CX001800, Boston Claude D. Pepper Older Americans Independence Center, NIA grant P30-AG031679, and NIA GEMSSTAR award R03AG060169.

Dr. Rosenberg: NIH NHLBI Patient-Oriented Career Development Award K23 HL127296 and NIH Loan Repayment Program L3O HL123413.

Dr. Driver: Veterans Affairs Merit Review Award Clinical Science R\&D I01CX000934-01A1.

The funders played no role in study design, collection, analysis, interpretation of data, writing of the report, or the decision to submit the paper for publication.
Compliance with Ethical Standards:

Conflict of Interest: The authors declare that they do not have a conflict of interest.

\section{REFERENCES}

1. Kremers MS, Hammill SC, Berul CI, et al. The National ICD Registry Report: Version 2.1 including leads and pediatrics for years 2010 and 2011. Heart Rhythm 2013;10(4):e59-e65.

2. Pokorney SD, Miller AL, Chen AY, et al. Implantable cardioverterdefibrillator use among Medicare patients with low ejection fraction after acute myocardial infarction. JAMA. 2015;313(24):2433-2440.

3. de Groot JR. Do elderly patients benefit from implantable-cardioverter defibrillators? Neth Hear J 2014;22(6):277-278.

4. Bibas $\mathbf{L}$, Levi $\mathbf{M}$, Touchette $\mathbf{J}$, et al. Implications of frailty in elderly patients with electrophysiological conditions. JACC Clin Electrophysiol 2016;2(3):288-294.

5. Kusumoto FM, Bailey KR, Chaouki AS, Deshmukh AJ, et al. Systematic review for the 2017 AHA/ACC/HRS Guideline for Management of Patients With Ventricular Arrhythmias and the Prevention of Sudden Cardiac Death: a report of the American College of Cardiology/American Heart Association Task Force on Clinical Practice Guidelines and the Heart Rhythm Society. Heart Rhythm 2018 Oct;15(10):e253-e274. doi: https://doi.org/10.1016/j.hrthm.2017.10.037.

6. Al-Khatib SM, Stevenson WG, Ackerman MJ, et al. 2017 AHA/ACC/ HRS Guideline for Management of Patients with Ventricular Arrhythmias and the Prevention of Sudden Cardiac Death. A Report of the American College of Cardiology/American Heart Association Task Force on Clinical Practice Guidelines and the Heart Rhythm Society. Heart Rhythm 2018 Oct;15(10):e190-e252. doi: https://doi.org/10.1016/j.hrthm.2017.10. 035.

7. Turakhia MP. Sudden cardiac death and implantable cardioverterdefibrillators. Am Fam Physician 2010;82(11):1357-1366.

8. Sharkey BJ. Functional vs chronologic age. Med Sci Sports Exerc 1987;19(2):174-178.

9. Merchant FM, Dickert NW Jr, Howard DH. Mandatory shared decision making by the centers for medicare \& medicaid services for cardiovascular procedures and other tests. JAMA. 2018;320(7):641-642. doi: https://doi.org/10.1001/jama.2018.6617.

10. Jensen TS CJ, Ashby L, Dolan D, Canos D, Hutter J. National coverage determination for implantable cardioverter defibrillators. https://www. cms.gov/medicare-coverage-database/details/nca-decision-memo.aspx? NCAId=288. Accessed April 4, 2019.

11. Lee SJ, Kim CM. Individualizing prevention for older adults. $J$ Am Geriatr Soc 2018 Feb;66(2):229-234. doi: https://doi.org/10.1111/jgs.15216.

12. Barra S, Providencia R, Paiva L, Heck P, Agarwal S. Implantable cardioverter-defibrillators in the elderly: rationale and specific age-related considerations. Europace. 2015;17(2):174-186.

13. Clegg A, Young J, Iliffe S, Rikkert MO, Rockwood K. Frailty in elderly people. Lancet. 2013;381(9868):752-762.

14. Fried LP, Tangen CM, Walston $\mathbf{J}$, et al. Frailty in older adults: evidence for a phenotype. J Gerontol A Biol Sci Med Sci 2001;56(3):M146-156.

15. Bibas $\mathbf{L}$, Levi $\mathbf{M}$, Touchette $\mathbf{J}$, et al. Implications of frailty in elderly patients with electrophysiological conditions. JACC Clin Electrophysiol 2016;2(3):288-294.

16. Moher D, Liberati A, Tetzlaff J, Altman DG; PRISMA Group. Preferred reporting items for systematic reviews and meta-analyses: the PRISMA statement. PLoS Med 2009;6(7):e1000097. doi: https://doi.org/10. 1371 /journal.pmed.1000097.

17. Rockwood $\mathbf{K}$, Song $\mathbf{X}$, MacKnight $\mathbf{C}$, et al. A global clinical measure of fitness and frailty in elderly people. CMAJ. 2005;173(5):489-495.

18. Kim DH, Kim CA, Placide S, Lipsitz LA, Marcantonio ER. Preoperative frailty assessment and outcomes at 6 months or later in older adults undergoing cardiac surgical procedures: a systematic review. Ann Intern Med 2016;165(9):650-660.

19. Buttery AK, Busch MA, Gaertner B, Scheidt-Nave C, Fuchs J. Prevalence and correlates of frailty among older adults: findings from the German health interview and examination survey. BMC Geriatr 2015;15(1):22.

20. Piotrowicz $\mathbf{K}$, Noyes $\mathbf{K}$, Lyness $\mathbf{J}$, et al. Physical functioning and mental well-being in association with health outcome in patients enrolled in the Multicenter Automatic Defibrillator Implantation Trial II [Multicenter Study; Randomized Controlled Trial; Research Support, Non-U.S. Gov't]. 
Eur Heart J 2007;28(5):601-607. doi:https://doi.org/10.1093/ eurheartj/ehl485

21. Fishbein DP, Hellkamp AS, Mark DB, et al. Use of the 6-min walk distance to identify variations in treatment benefits from implantable cardioverter-defibrillator and amiodarone: results from the SCD-HeFT (Sudden Cardiac death in heart failure trial). J Am Coll Cardiol 2014;63(23):2560-2568.

22. Kramer DB, Mitchell SL, Monteiro J, et al. Patient activity and survival following implantable cardioverter-defibrillator implantation: the ALTITUDE Activity Study. J Am Heart Assoc 2015;4(5)

23. Schernthaner C, Pichler M, Strohmer B. Lower body mass index and atrial fibrillation as independent predictors for mortality in patients with implantable cardioverter defibrillator. Croatian Med $J$ 2007;48(1):59-67.

24. Jahangir A, Mirza M, Shahreyar M, et al. Presence of obesity is associated with lower mortality in elderly patients with implantable cardioverter defibrillator. Int $J$ Obes 2018;42(2):169-174

25. Hsu JC, Varosy PD, Bao H, Wang Y, Curtis JP, Marcus GM. Low body mass index but not obesity is associated with in-hospital adverse events and mortality among implantable cardioverter-defibrillator recipients: insights from the National Cardiovascular Data Registry. J Am Heart Assoc 2012;1(6):e003863.

26. Zhan C, Baine WB, Sedrakyan A, Steiner C. Cardiac device implantation in the United States from 1997 through 2004: a population-based analysis. J Gen Intern Med 2008;23 Suppl 1:13-19.

27. Green AR, Leff $\mathbf{B}$, Wang $\mathbf{Y}$, et al. Geriatric conditions in patients undergoing defibrillator implantation for prevention of sudden cardiac death: prevalence and impact on mortality. Circ Cardiovasc Gual Outcomes 2016:9(1):23-30.

28. Gruenewald TL, Seeman TE, Karlamangla AS, Sarkisian CA. Allostatic load and frailty in older adults. J Am Geriatr Soc 2009;57(9):1525-1531.

29. Gostoli S, Bonomo M, Roncuzzi R, Biffi M, Boriani G, Rafanelli C. Psychological correlates, allostatic overload and clinical course in patients with implantable cardioverter defibrillator (ICD). Int $J$ Cardiol 2016;220:360-364

30. Bansal N, Szpiro A, Reynolds $\mathbf{K}$, et al. Long-term outcomes associated with implantable cardioverter defibrillator in adults with chronic kidney disease. JAMA Intern Med 2018.

31. Goldenberg I, Moss AJ, Hall WJ, et al. Causes and consequences of heart failure after prophylactic implantation of a defibrillator in the multicenter automatic defibrillator implantation trial II. Circulation 2006;113(24):2810-2817.
32. ATS Statement. Am J Respir Crit Care Med 2002;166(1):111-117.

33. DePew ZS, Karpman C, Novotny PJ, Benzo RP. Correlations between gait speed, 6-minute walk distance, physical activity, and self-efficacy in patients with severe chronic lung Disease. Respir Care 2013;58(12):21132119.

34. Peel NM, Kuys SS, Klein $\mathbf{K}$. Gait speed as a measure in geriatric assessment in clinical settings: a systematic review. J Gerontol A Biol Sci Med Sci 2013;68(1):39-46.

35. Abellan van Kan G, Rolland Y, Andrieu S, et al. Gait speed at usual pace as a predictor of adverse outcomes in community-dwelling older people an International Academy on Nutrition and Aging (IANA) Task Force. $J$ Nutr Health Aging 2009;13(10):881-889.

36. Tomiyama AJ, Hunger JM, Nguyen-Cuu J, Wells C. Misclassification of cardiometabolic health when using body mass index categories in NHANES 2005-2012. Int $J$ Obes 2016;40:883.

37. Hainer V, Aldhoon-Hainerová I. Obesity paradox does exist. Diabetes Care 2013;36(Suppl 2):S276-S281.

38. Stenholm S, Harris TB, Rantanen T, Visser M, Kritchevsky SB Ferrucci L. Sarcopenic obesity-definition, etiology and consequences. Curr Opin Clin Nutr Metab Care 2008;11(6):693-700.

39. McEwen BS. Allostasis and allostatic load: implications for neuropsychopharmacology. Neuropsychopharmacology. 2000;22:108.

40. Fava GA, Guidi J, Semprini F, Tomba E, Sonino N. Clinical assessment of allostatic load and clinimetric criteria. Psychother Psychosom 2010;79(5):280-284

41. Flint KM, Matlock DD, Lindenfeld J, Allen LA. Frailty and the selection of patients for destination therapy left ventricular assist device. Circ Heart Fail 2012;5(2):286-293.

42. Rowe R, Iqbal J, Murali-krishnan R, et al. Role of frailty assessment in patients undergoing cardiac interventions. Open Heart 2014;1(1):e000033.

43. Beattie JM, Kirkpatrick JN, Patton KK, Eiser AR. Hardwired for life? Implantable defibrillator dilemmas in older patients. Am $\mathrm{J}$ Med 2018;131(10):1143-1145.

Publisher's Note Springer Nature remains neutral with regard to jurisdictional claims in published maps and institutional affiliations. 